\title{
Diversidad de la vegetación durante su recuperación tras una creciente extraordinaria del Río Paraná Medio (Argentina)
}

\author{
Eduardo A. Franceschi ${ }^{1}$, Patricia S. Torres ${ }^{1} \&$ Juan P. Lewis ${ }^{2}$ \\ 1. Cátedra de Ecología, Facultad de Ciencias Agrarias-CIUNR, U.N.R. CC 14. S2125ZAA Zavalla, Santa Fe, Argentina; \\ efranceschi@arnet.com.ar, ptorres@citynet.net.ar \\ 2. Cátedra de Ecología, Facultad de Ciencias Agrarias, U.N.R. CONICET. CC 14. S2125ZAA Zavalla, Santa Fe, \\ Argentina; juanplewis@ciudad.com.ar
}

Recibido 04-V-2009. Corregido 08-XI-2009. Aceptado 01-XII-2009.

\begin{abstract}
Diversity of vegetation during its recovery from a catastrophic flood of the Medium Paraná River (Argentina). River floodplains have a high biological diversity that is intensely influenced by vegetation dynamics, changes in space and time scales, and the river's hydrological regime. The vegetation composition of the medium and lower Paraná River has been analyzed previously, with different approaches and criteria that cannot be compared. The aim of this study is to analyze the diversity of the herbaceous vegetation over long time spans, from its regeneration after a catastrophic flood to its recovery, in the North and South sites of the flooding valley of the Medium Paraná River. The first sampling of a pioneer community was performed after the 1982-83 catastrophic flood, and included the surveillance of two plots in two successive recovery stages. Floristic composition and cover-abundance of all species were recorded per plot. Floristic richness, using jackknife, Shannon and Hurlbert diversity and Simpson dominance curves were calculated for each site and for each survey, using EcoSim (software). Floristic richness was higher in the North of Medium Paraná, while dominance was higher in the South. Diversity indexes did not varied significantly and resulted in relatively stable values, because its components compensate each other. Rev. Biol. Trop. 58 (2): 707-716. Epub 2010 June 02.
\end{abstract}

Key words: River Paraná, floodplain, vegetation dynamics, EcoSim, diversity, floristic richness, regeneration.

Las planicies de inundación de algunos ríos son humedales que tienen una alta diversidad biológica cuya sustentabilidad puede ser amenazada por la regulación de los cursos de agua (Ward et al. 1999). Los tramos medio e inferior del Río Paraná comprenden una extensa planicie de inundación que no ha sido mayormente afectada por las represas instaladas en el Alto Paraná brasileño y el Paraná Encajonado (Matteucci et al. 2004). El problema de la diversidad ha sido abordado por diferentes autores, entre ellos Lewis et al. (1987), Zalocar de Domitrovic (1990, 1992, 1993), Franceschi \& Lewis (1991), Marchese \& Ezcurra de Drago (1992), Bó \& Malvárez (1999), Franceschi et al. 2000, que han analizado el problema con diferentes enfoques y métodos, en distintos grupos taxonómicos y escalas, que dificultan su comparación. Por su parte, Neiff (2001) ha brindado una visión general y crítica de la diversidad de los humedales sudamericanos.

La vegetación del río Paraná pertenece a la provincia fitogeográfica Paranaense (Martínez-Crovetto 1963, Cabrera 1976), su flora arbórea es de linaje tropical, relacionada al núcleo misionero de Prado \& Gibbs (1993), y se extiende hacia el sur por la planicie del río hasta el estuario del Plata. La vegetación de la planicie de inundación es predominantemente herbácea, excepto sobre albardones y bancos del cauce, donde es leñosa. En el punto más elevado del gradiente topográfico (albardones maduros) se encuentran bosques pluriespecíficos y, en el más bajo, sobre bancos del cauce, 
hay bosques en los que domina una especie (Tessaria integrifolia Ruiz et Pav. o Salix humboldtiana Willd.). Las comunidades herbáceas acuáticas tienen diferentes especies dominantes según el tipo de cuerpo de agua, y las palustres se caracterizan por la fuerte dominancia de una o pocas especies (Franceschi \& Lewis 1979, Franceschi et al. 1985). El conocimiento de la diversidad florística de estos humedales es importante para comprender la función de la vegetación en el ecosistema fluvial.

El régimen del Río Paraná presenta inundaciones anuales que, en períodos irregulares son más intensas y, con una frecuencia aproximada de dos por siglo, son extraordinarias con carácter catastrófico, ya que destruyen la vegetación herbácea y aún la leñosa, debido principalmente a la duración de la inundación, y en menor medida por erosión, sedimentación y remoción. Al descender las aguas, quedan grandes áreas descubiertas, donde se instala vegetación pionera que evoluciona hasta su estabilización. Este proceso ha sido estudiado en forma descriptiva, detectándose las tendencias generales de largo plazo, luego de la inundación catastrófica de 1982-83 hasta 2000-02 (Franceschi et al. 2000, Franceschi et al. 2005, Franceschi 2006). Sin embargo, la diversidad se ha descrito de forma puntual, y no comprende las etapas más avanzadas de recuperación de la vegetación en áreas ubicadas al norte y sur del Paraná Medio. Así, en la primera (norte), se utilizó la entropía de Brillouin para estimar la diversidad de las comunidades en 1984 (Lewis et al. 1987) y 1988 (Franceschi \& Lewis 1991); y en el área sur, el índice de Shannon para una serie de años entre 1986 y 1996 (Franceschi et al. 2000). Para dichos estudios, la superficie de la unidad muestral y el tamaño de la muestra fueron diferentes, y hasta el momento, no hay un análisis que utilice los mismos índices y que compare diferentes sitios a un mismo tiempo.

La diversidad florística está ligada a la dinámica de la vegetación, por lo que para su comprensión es necesario tener en cuenta las escalas espacial y temporal (Ward et al. 1999) y, localmente la altitud topográfica, que determina el gradiente hídrico, y el régimen de disturbios (nivel de inundación, pastoreo y fuego). El objetivo de esta contribución es analizar la diversidad de la vegetación herbácea a largo plazo, desde su reinstalación después de una inundación catastrófica hasta su recuperación, en dos áreas de inundación del valle en el Río Paraná.

\section{MATERIALES Y MÉTODOS}

Área de estudio: Se seleccionaron dos áreas en los extremos del Paraná Medio, provincia de Santa Fe, Argentina. Una en el norte (2813‘20” S y 59¹3‘08” W), próxima a El Rabón (ER), desde las cercanías del escarpe costero hacia el cauce principal. Allí predominan en el paisaje pajonales de Panicum prionitis Nees (paja), que son quemados frecuentemente como práctica de manejo de una ganadería primitiva. Otra en el sur (32 $16^{\circ} 21^{\prime \prime} \mathrm{S}$ y $\left.60^{\circ} 42^{\prime} 40^{\prime \prime} \mathrm{W}\right)$, en la isla Campo Rico (CR), reserva El Rico, en islas del cauce, desde el borde de una laguna central hacia el albardón periférico, donde por ser reserva natural no se practica ganadería ni se quema la vegetación, con predominio de pajonales de $P$. prionitis y cataisales de Polygonum acuminatum Kunth (catay).

Toma y análisis de los datos: Cuando el nivel del agua de la creciente catastrófica de 1982-83 disminuyó (Fig. 1) y fue posible acceder al terreno, se realizó el primer muestreo de la comunidad vegetal pionera $\mathrm{y}$, en años posteriores otros dos en etapas avanzadas de recuperación. Los años estudiados en ER fueron 1984, 1988 y 2002, y en CR 1986, 1988 y 2000. Los años corresponden cronológicamente a una etapa inicial, intermedia y madura del desarrollo de la vegetación después de la inundación extraordinaria. En ER se ubicaron a lo largo de $2 \mathrm{~km}, 19$ parcelas permanentes de $4 \times 4 \mathrm{~m}$ y en CR, 30 parcelas de $2.5 \times 2.5 \mathrm{~m}$ a lo largo de $600 \mathrm{~m}$. En cada parcela de ambas áreas se registraron las especies presentes y se estimó su abundancia-cobertura con la escala combinada de Braun-Blanquet (Braun-Blanquet 1979), que fue transformada a la escala numérica de van der Maarel (1979) previo al análisis estadístico. Los ejemplares recolectados están depositados en el Herbario de la Universidad Nacional de Rosario (UNR). 


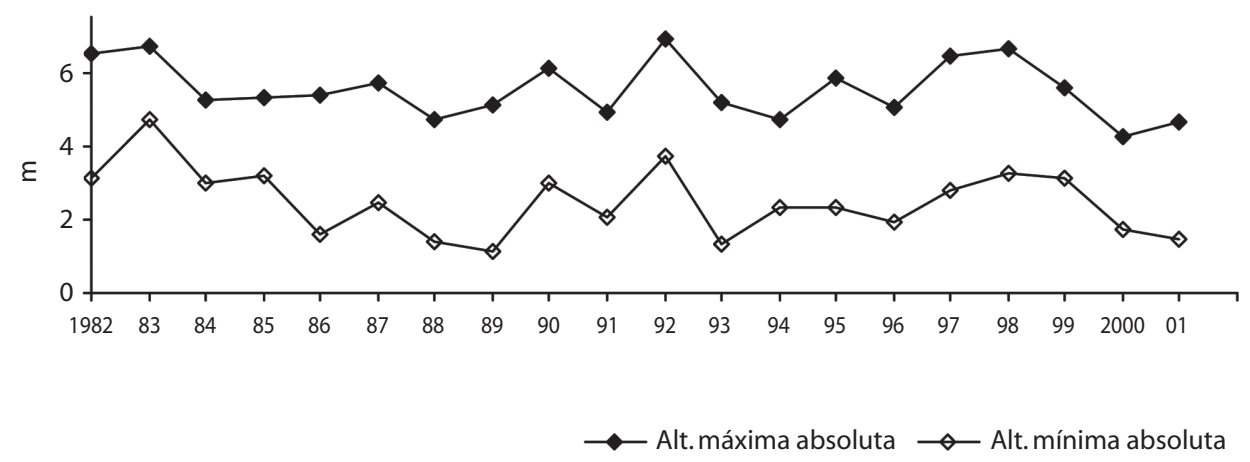

Fig. 1. Alturas hidrométricas del río Paraná en el Puerto de Diamante, Entre Ríos, Argentina (3204“08” S y 60³8‘22” W). Fuente: Dirección de construcciones portuarias y vías navegables, Puerto de Diamante.

Fig. 1. Water level of Parana river at Diamante Port, Entre Ríos, Argentina (3204’08” S and 60³8“22" W). Data from: Dirección de construcciones portuarias y vías navegables, Puerto de Diamante.

Se calcularon las curvas de riqueza florística, de diversidad de Shannon (H) y Hurlbert (PIE) y de dominancia de Simpson en ambas áreas para cada año, utilizando el programa EcoSim (Gotelli \& Entsminger 2007) basado en modelos de simulación. EcoSim, a través de un algoritmo, analiza al azar reiteradamente la muestra dada para diferentes tamaños de muestra. Este proceso permitió calcular no sólo la diversidad y sus componentes puntualmente (a través de un promedio), sino también construir curvas de las mismas. Estas curvas se pueden referir al área muestreada, por lo que es factible la comparación de dos muestras de diferente tamaño y/o con parcelas de distinta superficie.

Las fórmulas utilizadas para los índices de diversidad son las siguientes:

$$
\begin{gathered}
H=-\sum_{i=1}^{s} p_{i} \ln p_{i} \\
P I E=\left(\frac{N}{N-1}\right)\left(1-\sum_{i=1}^{s} p_{i}^{2}\right)
\end{gathered}
$$

siendo $\mathrm{p}_{\mathrm{i}}$ : la proporción de parcelas en la que está presente la especie i, s: número total de especies, ln: logaritmo natural y $\mathrm{N}$ : número total de parcelas.
Se calculó la riqueza florística usando jacknife (Manly 1998) en las dos áreas para los tres muestreos. Este valor es una estimación del número total de especies presentes en una comunidad, que es subestimado cuando se toma solamente una muestra de la misma. Se comparó la riqueza florística, diversidad de Shannon y de Hurlbert y dominancia de Simpson, en las dos áreas para los tres relevamientos, para la superficie total muestreada $\left(187 \mathrm{~m}^{2}\right)$ correspondiente al área menor (CR). Se calculó el número de especies exclusivas (especies registradas en un único año), para cada etapa en ambas áreas, ajustándolo a una superficie común para poder compararlos.

Se calculó el número de especies en cada clase de frecuencia, el promedio y desvío estándar del porcentaje de cobertura (BraunBlanquet 1979), para cada etapa y área.

\section{RESULTADOS}

La comparación de ambas áreas muestra que las curvas de los componentes de la diversidad en función de la superficie muestreada, producidas por el programa ECOSIM, presentan en la primera etapa (Fig. 2a), tanto para la riqueza florística como para la diversidad de Shannon, valores mayores en ER. El índice de diversidad de Hurlbert no muestra diferencias 
entre ambos sitios. Los valores del índice de dominancia son mayores en CR que en ER. En CR se observa una tendencia a la estabilización en la curva de riqueza florística, mientras que en ER no se encuentra la misma tendencia a la estabilización con el tamaño de muestra estudiado. En la segunda etapa (Fig. 2b), las variables se comportan en forma similar a la anterior aunque la dominancia en CR aumenta fuertemente. En la tercera y última etapa (Fig. 2c) las variables mantienen el mismo comportamiento, aunque desaparece la diferencia en la dominancia entre áreas.

En la primera etapa en ER se encontró un total de 124 especies y en CR 51 especies, siendo las estimaciones usando jacknife de 164 y 63 especies respectivamente (Fig. 3). En la segunda etapa la riqueza florística fue menor en ambas áreas, en ER se encontró un total de 77 especies y en CR 40, y las estimaciones con jacknife fueron de 100 y 54 especies respectivamente. En la tercera y última etapa, la riqueza florística vuelve a aumentar en ambas áreas: se registró un total de 94 especies en ER y 58 en CR. Las estimaciones con jacknife fueron de 130 y 74 especies respectivamente. El jacknife se aproxima más que otros métodos alternativos al valor verdadero de la riqueza florística (Palmer 1990, 1991), por lo que lo consideramos la mejor estimación de la misma.

La comparación de los componentes de la diversidad a lo largo de las tres etapas presenta, en las dos áreas estudiadas (Fig. 3), una riqueza florística con la misma tendencia: mayores valores en la primera etapa, mínimos en la segunda y nuevamente altos en la tercera, superando ligeramente en $\mathrm{CR}$ el valor de la primera etapa. La diversidad de Shannon tiene la misma tendencia encontrada en la riqueza florística; mientras que en la diversidad de Hurlbert la tendencia es similar pero casi imperceptible, pues los cambios a lo largo de las tres etapas son mínimos.

La dominancia es baja en la primera etapa, sube en la segunda (siendo muy alta en $\mathrm{CR}$ ), y en la última se mantiene en ER pero en CR baja hasta que se equiparan sus valores. En ambas áreas los máximos valores de la dominancia alcanzados en la segunda etapa, coinciden con el mínimo de riqueza florística y el mínimo de especies exclusivas. La proporción de especies exclusivas en cada etapa tiene igual tendencia en ambas áreas, con mínimos en la segunda etapa, pero en ER el máximo número de especies exclusivas se encuentra en la primera etapa mientras que en CR se da en la tercera.

En ambas áreas, el número de especies y la abundancia-cobertura promedio de las especies, en cada clase de frecuencia, tienen comportamiento inverso: el número de especies en cada clase de frecuencia es alto solamente en la clase más baja, con tendencia decreciente en las demás (Fig. 4a), mientras que los mayores valores de abundancia-cobertura se encuentran en las clases altas (Fig. 4b). O sea que hay muchas especies poco frecuentes y pocas especies muy frecuentes, si bien es en esta última clase donde se registran los valores más altos de abundancia-cobertura, que son los de las especies que dominan y caracterizan las comunidades.

\section{DISCUSIÓN}

El tema de la diversidad es motivo de controversia (Legendre et al. 2005, Tuomisto \& Ruokolainen 2006) y análisis (Orloci et al. 2002), y en los trabajos que lo abordan se encuentra una amplia heterogeneidad de mediciones, que dificultan y aún impiden, su comparación. La interpretación de las expresiones matemáticas de la diversidad, como carácter sintético de la comunidad, tiene fuertes limitantes si no se tienen en cuenta la escala espacial y temporal (Neiff 2001). Por otra parte, se conoce que la riqueza florística y, por lo tanto la diversidad, aumenta a medida que crece el tamaño de muestra hasta su estabilización, por lo tanto, si su estimación se reduce a un único valor, no se puede deducir en qué parte de la curva de la diversidad está situado. Esto hace que se pierda información, se dificulte la interpretación del patrón de diversidad y no se puedan comparar comunidades, dificultades que el programa EcoSim ayuda a superar. Todos los cálculos sobre la diversidad y sus componentes de este trabajo están referidos a la superficie total muestreada, por lo tanto no 
Riqueza florística

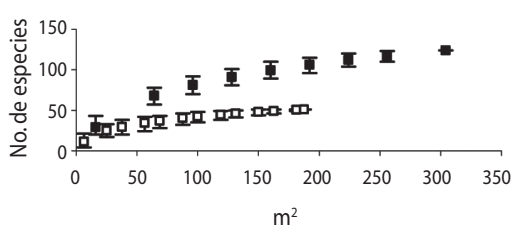

Diversidad de Shannon

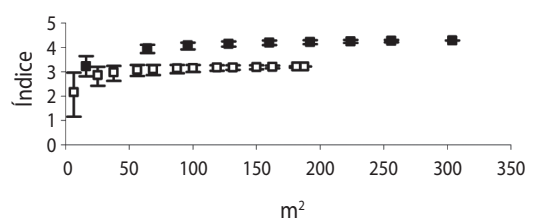

Dominancia

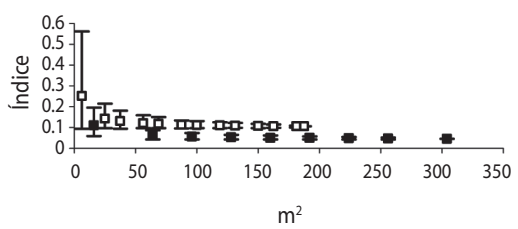

Diversidad de Hurlbert

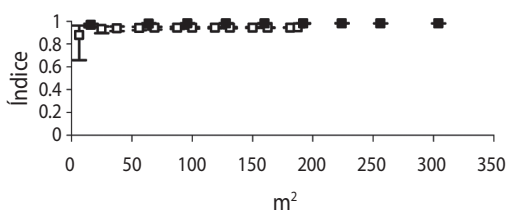

B. Etapa intermedia

Riqueza florística

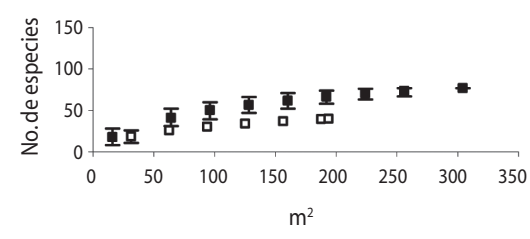

Diversidad de Shannon

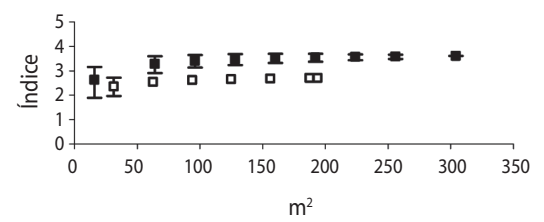

Dominancia

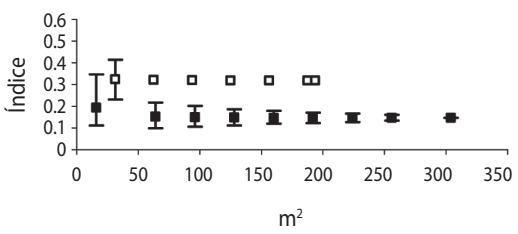

Diversidad de Hurlbert

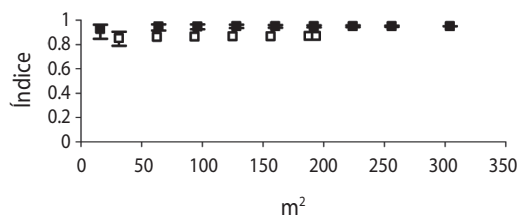

Fig. 2. Componentes de la diversidad en ER y CR según la superficie (EcoSim), y para las tres etapas evaluadas: A) inicial, B) intermedia y C) madura ( $\mathbf{\square}: \mathrm{ER}, \square: \mathrm{CR})$.

Fig. 2.Diversity components in ER and CR according to the sampled surface (EcoSim), and for the three evaluated stages: A) initial, B) intermediate and C) mature ( $\mathbf{\square}$ : ER, $\square:$ CR).

son comparables con las mediciones puntuales (promedios) de trabajos anteriores (Lewis et al. 1987, Franceschi \& Lewis 1991, Franceschi et al. 2000) de manera que el análisis con EcoSim mejora considerablemente la estimación de la diversidad y sus componentes.

La dinámica de la vegetación varía espacial y temporalmente, y en el río Paraná está 
Riqueza florística

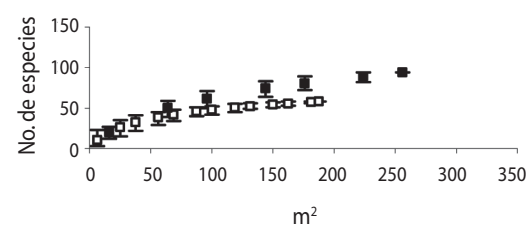

Diversidad de Shannon

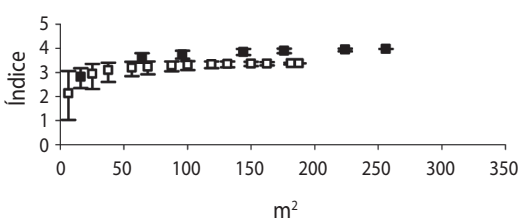

Dominancia

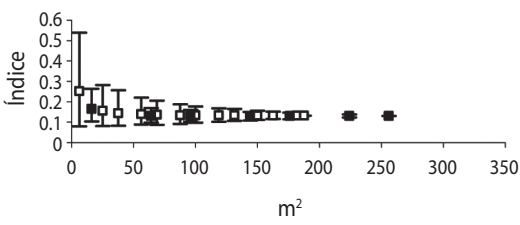

Diversidad de Hurlbert

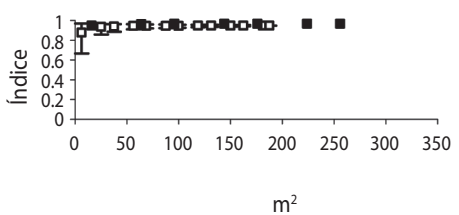

Fig. 2. Componentes de la diversidad en ER y CR según la superficie (EcoSim), y para las tres etapas evaluadas: A) inicial, B) intermedia y C) madura ( $\mathbf{\square}: \mathrm{ER}, \square: \mathrm{CR})$.

Fig. 2.Diversity components in ER and CR according to the sampled surface (EcoSim), and for the three evaluated stages: A) initial, B) intermediate and C) mature ( $\mathbf{\square}$ : ER, $\square$ : CR).

modelada en primera instancia por el régimen pulsátil del río (Franceschi 2006). Las comunidades vegetales se caracterizan por tener un alto número de especies de baja frecuencia (Braun-Blanquet 1979) y las comunidades herbáceas de la planicie de inundación del Paraná no son la excepción: un promedio del $65 \%$ de las especies se encuentra en la clase más baja de frecuencia (Fig. 4) y además muchas de estas especies son accidentales. Estas especies poco frecuentes inciden escasamente en la estructuración de la comunidad y no son consideradas para su caracterización (Carnevale \& Torres 1994), son las que suelen tener alta incidencia en los cálculos de diversidad. Por el contrario, son pocas las especies que presentan valores altos de abundancia-cobertura, como P. prionitis, Cynodon dactylon (L.) Pers., P. acuminatum y Ludwigia peploides (Kunth), que dominan y caracterizan las comunidades (Franceschi 2006). En general la diversidad varía con la latitud y en la cuenca del Plata es alta en latitudes bajas, como en el Pantanal y en el Paraná brasileño, y luego baja hasta el delta cisplatino donde vuelve a aumentar. Este comportamiento ha sido particularmente señalado en las formaciones leñosas de los albardones (Franceschi \& Lewis 1979, Menalled \& Adámoli 1995). La curva de riqueza florística en el área norte, en ER, no parece estabilizada, por lo que si se aumentara la superficie muestreada aumentaría el número de especies encontradas, siendo una aproximación el valor de jacknife de 164 especies. En el área sur, en CR, la curva de número de especies sí parece estabilizada (51 especies) y el tamaño de la muestra sería adecuado. Estos datos, si bien no permiten atribuir a las comunidades herbáceas una tendencia similar a la de los bosques, pues sólo dos áreas estudiadas son insuficientes para ello, tampoco la contradicen. Las diferencias entre los valores de riqueza florística encontrados entre ambas áreas estudiadas podrían atribuirse al gradiente térmico $\mathrm{N}-\mathrm{S}$, al mayor impacto de 
Riqueza florística

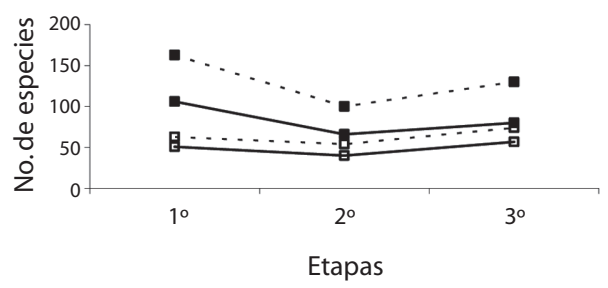

Diversidad de Hurlbert

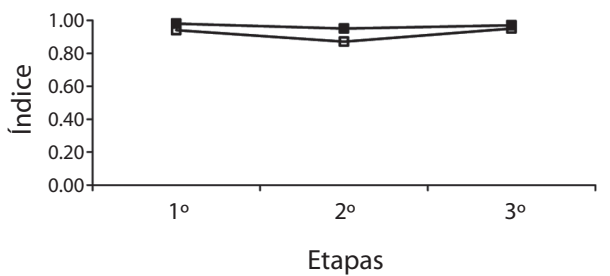

Especies exclusivas

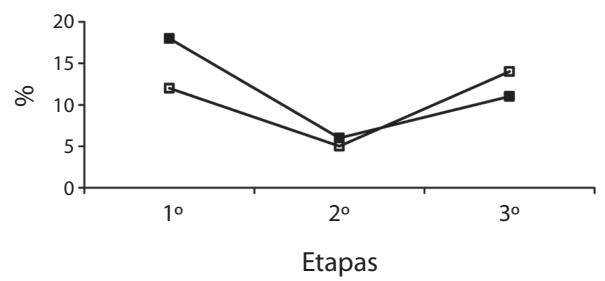

Diversidad de Shannon

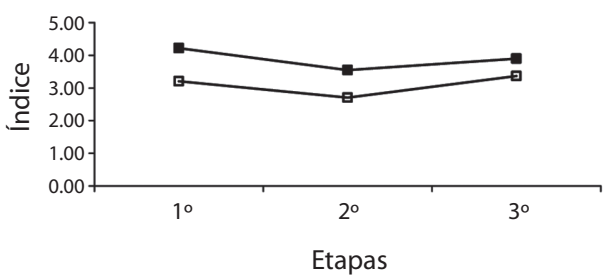

Dominancia

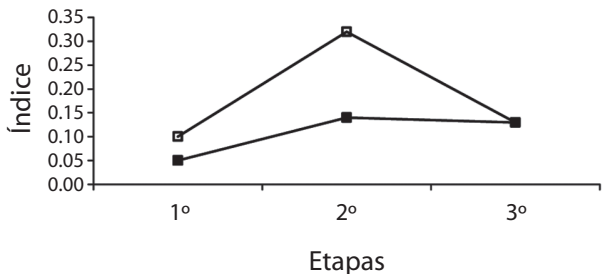

Fig. 3. Comparación de los componentes de la diversidad en todos los relevamientos y áreas estudiadas

$\mathrm{ER}, \square: \mathrm{CR}$, ----: jacknife).

Fig. 3. Comparison of diversity components during all surveys and sites studied ( $\square$ : ER, $\square$ : CR, ------: jacknife).

las crecientes y bajantes del río aguas abajo (la planicie de inundación se ensancha hacia el sur), a la diferente ubicación transversal de estas áreas dentro del valle y al fuego y pastoreo que en ER reducen la dominancia de $P$. prionitis favoreciendo a otras especies; además en CR parte de la superficie está ocupada por cataisales de Polygonum spp. que son comunidades con fuertes dominantes y menos especies que el pajonal (Franceschi et al. 1985), y no se encuentran en el área de trabajo de ER. También podría relacionarse con el papel que juega el río si actúa como corredor para la migración de las especies herbáceas, para lo cual serían necesarios trabajos adicionales.

En ER la vegetación pionera después de la inundación fue una comunidad integrada fundamentalmente por especies anuales y de corta vida, que eran especies poco frecuentes del pajonal maduro destruido. Esta comunidad resultó efímera, pues a medida que fueron recuperándose las poblaciones dominantes de los pajonales y gramillares, $P$. prionitis y $C$. dactylon respectivamente, sus especies se volvieron 


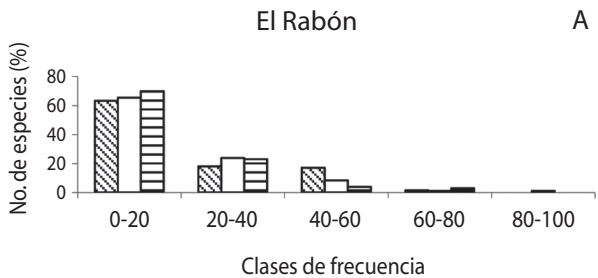

El Rico

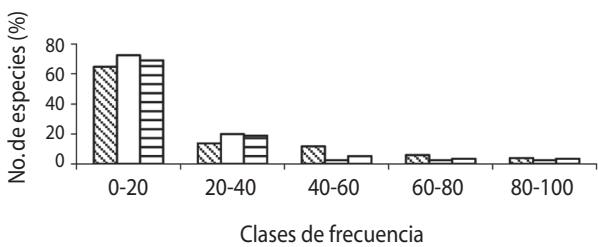

El Rabón B

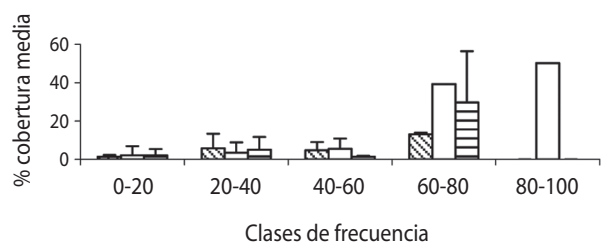

El Rico B

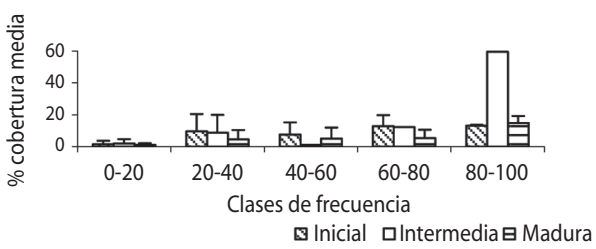

Fig. 4. Porcentajes del número de especies (A) y cobertura media (B), para cada clase de frecuencia, en ER y CR y las tres etapas analizadas.

Fig. 4. Percentages on species number (A) and cover average (B) for each frequency class, in ER and CR, and for three analysed stages.

raras y la comunidad perdió identidad (Franceschi et al. 2005). Este proceso está reflejado por un alto valor inicial de riqueza florística y especies exclusivas, y un valor mínimo de dominancia; mientras que en la segunda etapa, cuando la dominancia se concentra en escasas especies el índice de dominancia es máximo y mínima la diversidad. En CR la vegetación pionera estuvo constituida por incipientes pajonales de $P$. prionitis, y cataisales de Polygonum spp. con verdolagales de L. peploides (verdolaga) y canutillares de Panicum elephantipes Nees ex Trin. y Echinochloa P. Beauv. spp. (canutillos), vegetación similar a la destruida, que luego maduró (Franceschi et al. 2000). La riqueza florística de esta área es menor que la de ER durante todo el período estudiado; es mínima en la etapa intermedia y máxima en la final. Este aumento en el tercer muestreo puede relacionarse con la inundación de 1998 (Fig. 1), que eliminó muchas plantas de $P$. prionitis disminuyendo su dominancia, favoreciendo así la instalación de otras especies, que en su mayoría son exclusivas de este tercer estudio (Fig. 3). La promoción de la diversidad por el disturbio ya ha sido señalada en el caso de las inundaciones (Lewis et al. 1987, Franceschi et al. 2000). Deille et al. (2001) encontraron, en un río regulado y en comunidades leñosas, que la supresión de las inundaciones promueve la diversidad. Esta contradicción es sólo aparente, pues la supresión es un disturbio tanto o más importante que las inundaciones, además, en sectores aún sometidos a inundaciones si bien la riqueza florística es baja, la diversidad y equitatividad son altas debido a la disminución de la dominancia por las inundaciones.

Las crecientes catastróficas producen grandes áreas descubiertas y la diversidad en ellas es cero, puesto que la riqueza florística registrada es la expresión de los órganos aéreos de la planta, aunque una creciente, fuego o pastoreo pueden eliminar la parte aérea pero no la subterránea, permitiendo a muchas especies un rápido crecimiento del individuo, iniciándose un proceso de regeneración de la vegetación. 
Variaciones de menor magnitud en el nivel de las aguas, en ciclos anuales o de pocos años, que impactan en forma diferencial según el tramo del río y la posición dentro de la planicie de inundación, producen variaciones menos drásticas de la vegetación, como cambios en la abundancia de las especies y extinciones locales y temporales de las mismas (Franceschi 2006).

La vegetación herbácea, dispuesta en sectores de elevación topográfica intermedia entre albardones y bordes de cuerpos de agua, es particularmente susceptible a las variaciones de altura de aguas del río; en estas áreas las variaciones de la vegetación en el tiempo se deben más a diferencias cuantitativas de las especies más relevantes que a cambios en la composición florística, no ajustándose a un proceso sucesional, siendo el fenómeno más evidente en CR (Franceschi et al. 2000, Franceschi 2006).

Los valores de riqueza florística y dominancia reflejan este comportamiento, cuyos índices tienen tendencias casi opuestas, con variaciones de importancia solamente luego de crecientes extraordinarias. Los índices de diversidad no reflejan adecuadamente estas variaciones pues al compensarse entre sí sus componentes riqueza florística y dominancia dan como resultado valores relativamente estables al índice de diversidad: una única forma de medir la diversidad difícilmente será adecuada (Purvis \& Hector 2000). Los disturbios promueven la riqueza florística, como ya se dijo anteriormente, y disminuyen la dominancia, fenómenos que al compensarse entre sí restringen la variabilidad de los índices de diversidad reflejando la estabilidad de la vegetación a largo plazo. En síntesis la riqueza florística es mayor en el norte del Paraná Medio (ER), la dominancia lo es en el sur (CR) y los índices de diversidad no presentan variaciones amplias en las áreas y años considerados, pero sí sus componentes.

\section{AGRADECIMIENTOS}

A todos los que colaboraron en la toma de datos de campo. A CONICET y CIUNR por el apoyo financiero. A I. Barberis por la lectura crítica del manuscrito. A tres revisores anónimos y al Editor por sus comentarios.

\section{RESUMEN}

La diversidad florística en las planicies de inundación está ligada a la dinámica de la vegetación, por lo que su comprensión depende de las escalas espacial y temporal y el régimen hidrológico del río. La planicie de inundación del Río Paraná, Argentina, tiene una alta diversidad biológica, aolo conocida a través de estudios puntuales no comparables entre si. El objetivo de esta contribución fue analizar la diversidad de la vegetación herbácea a largo plazo, desde su reinstalación después de una inundación catastrófica hasta su recuperación, en dos áreas (norte y sur) del Paraná Medio. Cuando el nivel del agua disminuyó después de la creciente catastrófica de 1982-83, se realizó el primer muestreo en una comunidad pionera, y en años posteriores, otros dos en etapas avanzadas de recuperación de la vegetación. Se registraron las especies presentes y se estimó su abundanciacobertura. Se calcularon las curvas de riqueza florística, de diversidad de Shannon y Hurlbert y de dominancia de Simpson en ambas áreas, utilizando el programa EcoSim. La riqueza florística resultó mayor en el norte del Paraná Medio, mientras que la dominancia lo fue en el sur. Los índices de diversidad no reflejan estas variaciones, pues al compensarse entre sí sus componentes, dan como resultado valores relativamente estables.

Palabras clave: Río Paraná, planicie de inundación, dinámica de la vegetación, EcoSim, diversidad, riqueza florística, regeneración.

\section{REFERENCIAS}

Bó, R.F. \& A.I. Malvárez. 1999. Las inundaciones y la biodiversidad en humedales. Un análisis del efecto de eventos extremos sobre la fauna silvestre, p. 169184. In A.I. Malvárez (ed.).Tópicos sobre humedales subtropicales y templados de sudamérica. UNESCO. Montevideo, Uruguay.

Braun-Blanquet, J. 1979. Fitosociología. H. Blume, Madrid, España.

Cabrera, A. 1976. Regiones Fitogeográficas Argentinas. ACME, Buenos Aires, Argentina.

Carnevale, N.J. \& P.S. Torres. 1994. Las especies vegetales raras y su volumen de hábitat en Santa Fe, Argentina. Rev. Biol. Trop. 42: 81-88.

Deiller, A.F., Walter, J.M.N. \& M. Trémolierès. 2001. Effects of flood interruption on species richness, diversity and floristic composition of woody 
regeneration in the Upper Rhine alluvial hardwood forest. Regulated Rivers: Research \& Management 17: 393-405.

Franceschi, E.A. \& J.P. Lewis. 1979. Notas sobre la vegetación del valle santafesino del Río Paraná (R.A.). Ecosur 6: 55-82.

Franceschi, E.A. \& J.P. Lewis. 1991. Early stages in the Parana river tall grassland recovery after an extraordinary flood. Coenoses 6: 47-32.

Franceschi, E.A. 2006. Regeneración de la vegetación herbácea luego de una inundación catastrófica en la llanura de inundación del Río Paraná. Tesis Doctoral, Universidad Nacional de Rosario, Rosario, Argentina.

Franceschi, E.A., D.E. Prado \& J.P. Lewis. 1985. Comunidades vegetales y mapa de vegetación de la Reserva El Rico e islas aledañas, Provincia de Santa Fe. Rep. Argentina. Universidad Nacional de Rosario, Rosario, Argentina.

Franceschi, E.A., P.S. Torres, D.E. Prado \& J.P. Lewis. 2000. Disturbance, succession and stability: a ten year study of temporal variation of species composition after a catastrophic flood in the river Paraná, Argentina. Community Ecol. 1: 205-214.

Franceschi, E.A, P.S. Torres \& J.P. Lewis. 2005. Recovery and stability of Paraná river floodplain grasslands twenty years after a catastrophic flood. Community Ecol. 6: 209-217.

Gotelli, N.J. \& G.L. Entsminger. 2007. EcoSim: Null models software for ecology. Version 7. Acquired Intelligence Inc. \& Kesey-Bear. Jericho, VT 05465. (Disponible en línea http: //garyentsminger.com/ecosim.htm)

Legendre, P., D. Bocard, \& P.R. Peres-Neto. 2005. Analyzing beta diversity: partitioning the spatial variation of community composition data. Ecol. Monogr. 75: 435-450.

Lewis, J.P., E.A. Franceschi \& D.E. Prado. 1987. Effects of extraordinary floods on the dynamics of tall grasslands of the river Paraná valley. Phytocoenologia 15: 235-251.

Manly, B.F.J. 1998. Randomization, bootstrap and Monte Carlo methods in biology. Chapman \& Hall, Londres, Inglaterra.

Marchese, M. \& I. Ezcurra de Drago. 1992. Benthos of the lotic environments in the Middle Paraná river system: transverse zonation. Hydrobiologia 237: 1-13.

Martínez-Crovetto, R. 1963. Esquema fitogeográfico de la provincia de Misiones (República Argentina). Bonplandia 1: 171-223.
Matteucci, S., J. Morello, A. Rodríguez \& N. Mendoza. 2004. El alto Paraná encajonado argentino-paraguayo: mosaicos de paisaje y conservación regional. FADU-UNESCO, Buenos Aires, Argentina.

Menalled, F.D. \& J.M. Adámoli. 1995. A quantitative phytogeographic analyses of species richness in forest communities of the Paraná river delta, Argentina. Vegetatio 120: 81-90.

Neiff, J.J. 2001. Diversity in some tropical wetland systems of South America, p. 157-186. In W. Gopal, J. Junk \& J.A. Davis (Eds.). Biodiversity in wetlands: assessment, function and conservation. Vol. II. Backhuys, Leiden, Holanda.

Orlóci, L., M. Anand \& V.D. Pillar. 2002. Biodiversity analyses: issues, concepts, tecniques. Community Ecol. 3: 217-236.

Palmer, M.W. 1990. The estimation of species richness by extrapolation. Ecology 71: 1195-1198.

Palmer, M.W. 1991. Estimating species richness: the second-order jacknife reconsidered. Ecology 72: 1512-1513.

Prado, D.E. \& P. Gibbs. 1993. Patterns of species distribution in the dry seasonal forests of South America. Ann. Missouri Bot. Gard. 80: 902-927.

Purvis, A. \& A. Hector. 2000. Getting the measure of biodiversity. Nature 405: 212-219.

Tuomisto, H. \& K. Ruokolainen. 2006. Analyzing or explaining beta diversity? Understanding the targets of different methods of analysis. Ecology 87: 2697 2708 .

van der Maarel, E. 1979. Transformation of cover-abundance values in phytosociology and its effects on community similarity. Vegetatio 39: 97-114.

Ward, J.V., K. Tockner \& F. Schiemer. 1999. Biodiversity of floodplain river ecosystems: ecotones and connectivity. Regulated Rivers: Research \& Management 15: 125-139.

Zalocar de Domitrovic, Y. 1990. Efecto de las fluctuaciones del nivel hidrométrico sobre el fitoplancton en tres lagunas isleñas en el área de la confluencia de los ríos Paraná y Paraguay. Ecosur 16: 1-23.

Zalocar de Domitrovic, Y. 1992. Fitoplancton de ambientes inundables del río Paraná (Argentina). Revue d'Hydrobiologie Tropicale 25: 175-186.

Zalocar de Domitrovic, Y. 1993. Fitoplancton de una laguna vegetada por Eichhornia crassipes en el valle de inundación del río Paraná (Argentina). Ambiente Subtropical 3: 39-67. 